\title{
Evaluation of Conventional methods for the Detection of Lesions in Diabetic Retinopathy Images: A Research
}

\author{
R.Ravindraiah, S.Chandra Mohan Reddy
}

\begin{abstract}
Diabetes Mellitus (DM) is a sporadic ailment which arises with the vagaries in blood glucose levels. Prolonged history of DM will result in retinal vasculature impediments and leads to Diabetic Retinopathy (DR). The patho is characterized by leakage of blood, fat and protein based particles into the macula and instigates the vision problems. The reliability Conventional clinician's screening methods is dependent on skilled professionals for diagnosis and screening. It costs to a great deal of time with manual labor and hence there is a great need to automate DR detection. Usage of image processing and machine learning approach to sense various retinopathy aberrations gained huge attraction in recent past. This paper reveals various DR detection and classification methods, including tools, implemented techniques and datasets used. It wishes to help researchers by giving brief literature review of merits and demerits of existing methods, so that it will help them to plan future developments.
\end{abstract}

Key words-Diabetes Mellitus (DM), Diabetic Retinopathy (DR), Retinal lesions

\section{INTRODUCTION}

Diabetic Retinopathy (DR) is a result of prolonged history of type 1 and type 2 diabetes[1-2]. DR is principally characterized as Non-Proliferative DR (NPDR) and Proliferative DR (PDR) [3-4]. It is asymptotic in the initial stages (NPDR) and its victims may get affected with blurred vision, floaters in the chronic stages (PDR) and may even lead to permanent vision loss. NPDR is characterized by the growth of microaneurysms, haemorrhages and blood capillaries get swollen and may eventually blocked. New blood vessels then proliferates to nourish the retinal tissue. These blood vessels are weak and fragile and may get ruptured leaking blood, protein and fat particles into the retinal fundus. These exuded particles get accumulated as soft and hard exudates. Figure 1 represents a typical DR fundoscope image which represents the significant retinal lesions and figure 2 represents the normal and distracted vision as a result of exudate sediments within the retinal fundus. This paper presents a critical review of various dedicated research contributions for the detection and classification of retinal lesions in the DR images.

Revised Version Manuscript Received on July 18, 2019.

R.Ravindraiah, Research Scholar, Department of ECE, JNT University Ananthapuramu, Anantapuramu, Andhra Pradesh, India, (email: ravindra.ranga@gmail.com)

S.Chandra Mohan Reddy Associate Professor, Department of ECE, JNT University Ananthapuramu, Ananthapuramu, Andhra Pradesh, India (Email: email2cmr@gmail.com)

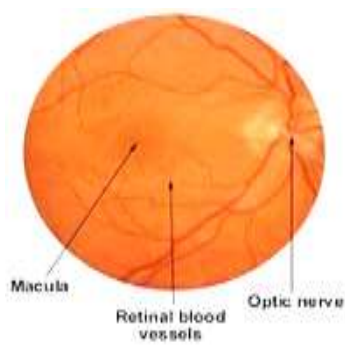

(a)

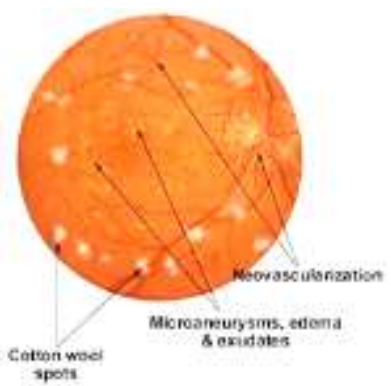

(b)
Figure 1: (a) Normal retina; (b) DR Fundus Image [Image Courtesy: Medical Eye Center, Oregon]

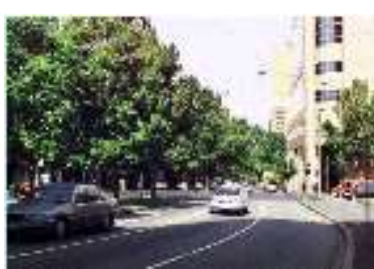

(a)

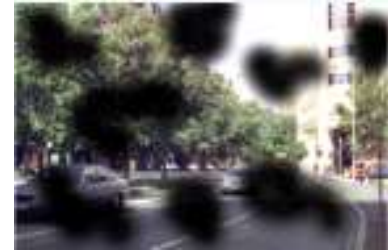

(b)
Figure 2: Vision with and without Diabetic Retinopathy.

\section{LITERATURE REVIEW OF EXISTING METHODS \& RESULTS}

Image segmentation aims to separate homogenous and meaningful regions and are widely used in industrial, military

and medical applications. DR detection systems are been using one or more following methods which includes: Morphological, Clustering, Classifier Thresholding, Markov random fields, Artificial Neural Networks (ANN), Deformable models, Atlas guided approach, edge and region based segmentation. The following literature represents various works developed for analyze DR characteristic that are bound to any one or more methods that specified above [5].

Phillips.R.P et al. [6] proposed a computerized DR detection system to assess repeatability, reproducibility and accuracy values by using. Images are sharpened and allowed to global thresholding algorithms to detect hard and soft exudates respectively. A disparity of $3 \%$ to $17 \%$ of repeatability and reproducibility parameters for hard and soft exudates is observed. And a mean sensitivity of $87 \%$ is 


\section{EVALUATION OF CONVENTIONAL METHODS FOR THE DETECTION OF LESIONS IN DIABETIC RETINOPATHY IMAGES: A RESEARCH}

reported. This method is fit for drusen and bare sclera detection, but fails to detect CWS. Gardner G.G et al., [7] trained Back propagation Neural Network (NN) classifier using 147 DR and 32 normal retinal images for to characterize lesion features against ophthalmologist resolution. Using Receiver Operator Coefficient (ROC) curves they achieved mean sensitivity and specificity values of $88.40 \%$ and $83.50 \%$ respectively on $200 \mathrm{DR}$ and 101 normal fundus retinal images. Ege et al. [8] addressed Bayesian classifier to find the disparity between cotton wool spots and exudates and reported a mean sensitivity and specificity of 52\% and $62 \%$ respectively. Wang. H et al. [9] used of Minimum Distance Discriminant (MDD) classifier for DR classification. At first, they used a brightness transform function to correct the illumination effects in the images. With the effects of multiple noises this method is led to misclassification of abnormal images Sinthanayothin. C et al. [10], [41], introduced a new technique called Moat operator and combined with a window-based recursive region growing algorithm to extract features of NPDR. They used a small dataset of 30 images of which 21 are with exudates and other are normal images and accomplished sensitivity and specificity of $88.5 \%$ and $99.7 \%$ respectively. They concluded that this method is well fit for to detect hard exudates than faint lesions. Walter.T et al. [11] performed morphological reconstruction for lesion detection and reported a mean sensitivity of $92.8 \%$ and predictive value of $92.4 \%$ respectively on a small dataset of 30 images. Osareh A. et al. [12] used features extracted from FCM segmentation and performed evaluation of five classifiers (Linear Delta Rule, K-Nearest Neighbors, neural networks (NN) and quadratic Gaussian classifier). They concluded that performance of NN classifier and Snake morphology is better compared to other methods. They reported a maximum accuracy of $90.1 \%$ for exudate detection. This method fails to detect faint exudates. Sinthanayothin C et al. [13] suggested the use of highest intensity variation for OD detection and multilayer perception neural network for blood vessels identification and spotted hard exudates using a recursive region growing technique. An average sensitivity of $80.21 \%$ and specificity of $70.66 \%$ is attained by using 283 DR images and 484 normal images. Osareh A et al. [14] fed features from coarse FCM clustering method to NN classifier and attained sensitivity and specificity of $93 \%$ and $94.10 \%$ respectively. The usage of FCM makes the system sensitive to noise and is failed to detect pale lesions. Sanchez C.I et al. [15] applied Kirsch operator to detect exudates by using features like color, edge sharpness and geometric features. They reported a sensitivity of $79.62 \%$ on a private dataset of 20 images and concluded that their system fails to detect few exudates from blood capillaries.

Usher D et al. [16] demonstrated an Artificial Neural Network (ANN) based classification and reported a variable sensitivity/ specificity pairs as $95.1 \% / 46.3 \%, 70.8 \% / 78.9 \%$, 94.8\%/ 52.8\%. Grisan et al. [17] applied Linear Discriminative Analysis (object classification) and Bayesian MAP classifier (pixel classification) and) and achieved a sensitivity of $0.83,0.71,0.73$, and a specificity of $0.94,0.99$, 0.91 for hard exudates, haemorrhages, cotton wool spots (CWS) respectively on 200 retinal images. Their method suffers from distinguishing faint haemorrhages from pigmentation variation. Zhang X et al. [18], performed SVM classification for DR grading. In the pre step a three-step bottom-up means and segmented them using a two-step improved FCM in Luv color space is used for bright lesion detection and feature extraction. Fleming A.D. et al. [19] identified target lesions via multi-scale morphological processing approach using local properties. A binary decision is carried out through the use of thresholding. Sensitivity and specificity of $95.0 \%$ and $84.6 \%$ is obtained on attest set of 13219 images of which exudates are contained in 300 images. Niemeijer M et al. [20] tested 300 images using k-nearest neighbor $(\mathrm{kNN})$ and linear discriminant classification methods for to discriminate various retinal lesions. On a test set of a sensitivity/specificity of $0.95 / 0.86$ is reported. Giri Babu Kande et al. [21] detected exudates using spatial weight induced standard Fuzzy C means (FCM) clustering method and reported sensitivity and specificity of $86 \%$ and $98 \%$ respectively on STARE and DIARETDB1 datasets. This system lacks in robustness as convention FCM uses Euclidean distance metric that is prone to outliers' problem. Sanchez C.I et al. [22] applied color, brightness features to Fisher's linear discriminant method for bright lesion detection. This method is limited with misclassification of faint exudates from hard exudates and lacks clinical validation due to the usage of small database. On a dataset of 58 images a mean sensitivity of $88 \%$ is reported. Acharya U.R et al. [23] used non-linear Higher Order Spectra (HOS) to features like shape and contours variation and are applied to SVM for classification. Using 300 subjects a mean sensitivity and specificity of $82.5 \%$ and $88 \%$ is attained. Sopharak.A et al. [24] employed a sequence of morphological and thresholding methods and conveyed a mean sensitivity and specificity of 80 of $99.46 \%$ respectively using 40 subjects. This method fails to distinguish the blood vessels from exudates which poses contrast similarities. They used a specific software for validating the results and hence lag clinical validation. They experimented with a naïve bayes classifier by amassing the features count in progressive stages in [25]. Sensitivity, specificity, precision and accuracy values are assessed as $93.38 \%, 98.14 \%, 47.51 \%$ and $98.05 \%$ respectively. Suthammanas J et al. [26] introduced Retinal Thickness Analyzer (RTA) to aid tele screening method for DR classification. A mean sensitivity of $93 \%$ is reported. Acharya U.R et al. [27] fed many features extracted using morphological methods and applied to SVM classifier for retinal lesion classification. On a dataset of 331 fundus images they attained $82 \%$ of sensitivity and $86 \%$ specificity. Sopharak et al. [28] experimented a mix of morphology, FCM and nearest neighbor DR classification. This method suffers from additive noise and fails to detect pale exudates and using a small dataset of 10 images a mean sensitivity and specificity of $87.3 \%, 99.3 \%$ are achieved. A comparative evaluation of three neural network classifiers (Support Vector Machine (SVM), Multilayer perceptron (MLP), Radial basis function $(\mathrm{RBF})$ ) for to investigate presence of DR in fundoscope images is done by Garcia M et. al., [29]. A mean sensitivity Blue Eyes Intelligence Engineering 
and Positive Predictive value as $88.14 \%, 80.72 \%$ for MLP, $88.49 \%, 77.41 \%$ for RBF and $87.61 \%, 83.51 \%$ for SVM respectively is reported on a dataset of 117 images.

Garcia et al. [30] exploited a string of global, local, and adaptive threshold methods to detect the exudates presence. At first, green and luminosity components are used to enhance images contrast. Radial basis classifier is used to discriminate the target lesions from background. On a dataset of 117 images they achieved $92.01 \%$ of Sensitivity and $86.4 \%$ of positive predictive value. Sopharak et al. [31] localized OD using highest entropy feature and segmented blood vessel using decorrelation stretch and thresholding followed with target lesion detection using FCM method. A mean sensitivity and specificity of $92.18 \%$ and $91.52 \%$ are achieved using a local dataset of 40 images and are validated ophthalmologists hand drawn ground truth image. Sanchez et al. [32] applied mixture models (semi-parametric statistical technique) based dynamic threshold approach. For DR detection. Exudates and cotton wool spot are discriminated using feature like high intensity, edges sharpness. On a dataset of 80 images a mean sensitivity and specificity of $92.02 \%$ and $96.8 \%$ respectively are attained. They suggested the use of 2-D Markov Random Field (MRF) models at the expanse of spatial correlation. Osareh A et al. [33] fed the features from FCM coarse segmentation to ANN for DR classification and assessed sensitivity, specificity and predictivity values as $96.0 \%$, $94.6 \%$ and $92.1 \%$ respectively using 300 images. This system works well for only Luv color space and lacks in detection of faint lesion. A coarse and fine segmentation of non-dilated retinal images using FCM and morphological techniques is proposed by Sopharak. A. et al. . [34]. They validated the results using ophthalmologists' hand-drawn ground-truths and attained $87.28 \%$ of sensitivity, $99.24 \%$ of specificity and $99.11 \%$ of accuracy. This method fails to detect some faint exudates and unable extract few exudates as the images are affected with noise and other imaging artefacts. Welfer $e t$ al. [35] verified a coarse to fine exudate detection using morphological and H-maxima transform in Luv color space and achieved a mean sensitivity and specificity of $70.48 \%$ and $99.84 \%$ is assessed on DIARETDB1 (89 images) database. They concluded that their method achieved little specificity values for non DR images. Sopharak.A, et al. [36] did comparative evaluation of FCM vs morphological approach used in their previous work [34]. They attained greater accuracy through morphological reconstruction than FCM. The end results are validated using ophthalmologist's ground truth images and reported sensitivity, specificity and accuracy of $88.1 \%, 99.2 \%$ and $99 \%$ respectively. Dupas B et. al. [37] used 115 images to grade DR using mathematical morphological operations and K-Nearest neighbor classifier (KNN) and accessed $92.8 \%$ of sensitivity and $92.4 \%$ of positive predictive value. Sanchez. C et al. [38] incorporated spatial correlation with high-level contextual features and classified DR images through linear discriminant classifier. This approach is unsuccessful in identifying pale and few bright lesions. On a dataset of 72 images an average of $91 \%$ sensitivity and $70 \%$ specificity values are attained. Agurto et al., [39] tested MESSIDOR database with a novel Multiscale Amplitude Modulation (AM) and Frequency Modulation (FM) methods to discriminate normal and DR images. This article assessed a figure of 0.98 receiver operating characteristic (ROC) value.

Abramo .M.D et al. [40] introduced innovative approach for DR detection called EyeCheck algorithm and assessed a value of $83.9 \%$ accuracy in detecting numerous retinal lesions. S. Kavitha et al. [42] attained the disparity in exudates from the backgroun pixels using a non-linear diffusion based segmentation method. The performed binarization and mathematical morphology in CIE Lab color space and applied thresholding on color histogram to distinguish hard and soft exudates. They reported of sensitivity, specificity and accuracy as $89.78 \%, 99.12 \%$ and $99.07 \%$ respectively on DIARETDB1 dataset. Akram [43] suggested Gabor wavelet, multilayered thresholding methods are used to segment blood capillaries followed by OD localization using average filter and thresholding methods. And a hybrid fuzzy classifier is used to spot bright and dark lesions. Using 290 images taken from DiaretDB0, DiaretDB1, STARE and DRIVE databases an accuracy of $93.71 \%$ is obtained. Rocha et al. [44] constructed a visual word dictionary using Speeded up Robust Features (SURF), Scale Invariant Feature Transform (SIFT), and k-means clustering to identify red and bright lesions. They attained an area under curve (AUC) of 0.88 on DiaretDB1 (89) image data set. Sharib ali et al. [45] generated an atlas image to detect lesion candidates by thresholding it. The input images are wrapped onto atlas co-ordinates to obtain the atlas image. They tested HEI-MED database (169 images) of and attained an accuracy of $83.0 \%$. Harangi.B et al. [46] demonstrated retinal lesion classification using an Active Contour Model (ACM) and adaptive boosted Naïve Bayes classifier and reported a mean sensitivity and specificity of $75.76 \%$ and $91.64 \%$. In [47] they used morphological techniques is used to detect candidate lesions and accomplished lesion's near boundary by using a sparse field algorithm as a level set method for the minimization of ChanVese energy function. On DIARETDB1 dataset they attained an average of $79.82 \%$ sensitivity and $95.64 \%$ specificity. They concluded that ACM model do not produce optimum results in detecting exudates contours. They extended their work in [48] by labeling the candidates as true or false exudates and attained an average sensitivity and Positive predictive value as $86 \%$ and $84 \%$ respectively on HEI-MED and DIARETDB 1 public datasets. Zhang et al. [49] suggested the use of morphological operators to estimate lesion sections. Then a lesion based classification is applied with classical and contextual features to remove the false positives from lesion map. Pereira et al. [50] combined thresholding and Ant Colony Optimization for DR detection and concluded that their method produced superior performance than conventional Kirsch filter. They reported a mean sensitivity, specificity and positive predictive values as $80.82 \%, 99.16 \%$ and $73.01 \%$ respectively on HEI-MED dataset. Medhi J.P. N et al. [51] normalized the retinal images in RGB, YIQ and HIS color space and 


\section{EVALUATION OF CONVENTIONAL METHODS FOR THE DETECTION OF LESIONS IN DIABETIC RETINOPATHY IMAGES: A RESEARCH}

segregated OD and blood vessels using morphological operators and ostu thresholding. Then the exudates are extracted by applying logical AND operation on Hue image and saturation plane. They attained a mean sensitivity of $77.73 \%$ and Specificity of $98.72 \%$ by using a total of 1374 images taken from DIARETDB1, MESSIDOR, DRIVE and HRF public databases.

Harangi.B et al. [52] extracted features obtained from a sequence preprocessing steps and performed image and lesion level classification using Baye's classifier. This article stated an image level sensitivity and specificity values as 0.92 and 0.68 (on DIARETDB1 database) and 0.87 and 0.86 (on HEI-MED database) and lesion based sensitivity of 0.86 on DIARETDB1 database. Imani.E et al. [53] developed novel Morphological Component Analysis (MCA) method to distinguish exudates from blood capillaries and attained a final exudate map using mathematical morphology and dynamic threshold methods. An accuracy of 0.961, 0.948 and 0.937 assessed on DiaretDB (89 images), HEI-MED (169 images) and e-ophtha (82 images) datasets respectively. Shuang Yu et al. [54] detected DR lesions morphologically and classified them using convolutional neural networks (CNN) classifier. They attained sensitivity, specificity and high pixel wise accuracy of $88.85 \% \quad 96 \%$ and $91.92 \%$ respectively on E-Optha public database (47/82 exudate images). Sil Kar. S et al. [55] detected blood vessels and OD using Kernel induced Fuzzy C Means clustering and morphological operators respectively. Bright and dark lesions are detected using Differential Evolution algorithm and morphological based approach. Matched Filtering and Laplacian of Gaussian Filtering (LoG) for bright and dark lesions detection and Mutual Information Maximization method to distinguish them. An average accuracy of $97.71 \%$ is obtained on randomly selected $50 \%$ images from each of the DIARETDB1, STARE, DRIVE and ROCh databases. Also they used 300/1200 images from MESSIDOR database. In [56] they opted a modified Fuzzy entropy maximization method for vessel segregation followed by matched filtration and LoG thresholding to grade DR by identifying various retinal lesions. They increased the dataset size i.e., 95\% images of each of DIARETDB1, STARE, DRIVE databases and $94 \%$ images of MESSIDOR database and attained sensitivity/ specificity of $93.14 \% / 93.19 \%$ for bright lesion detection and $94.01 \% / 93.58 \%$ for dark lesion detection. Gao Z et al. [57] addressed a deep convolutional neural network based DR classification system and achieved an average accuracy of $88.72 \%$ using the public datasets like DIARETDB0, DIARETDB1, DRIVE and STARE.

\section{CONCLUSION}

DR is startling health issue causing vision impediments if deserted or not well treated. It signifies the failure of retinal function. Implementation of digital image processing methods can enhance the conventional medical imaging systems and assists the clinicians for easy diagnosis of the patho. A brief description of existing methods that contributed for the detection of multiple features in DR images are been presented in this paper. These works includes usage of various preprocessing steps to enhance the details of
DR images in multiple color spaces followed with localization of OD, removal of blood vessels and detection of candidate lesions. Most of works used publically available databases like ], Digital Retinal Images for Vessel Extraction (DRIVE) [58], STructured Analysis of the Retina (STARE) [59], Diaretdb0 [60], Diaretdb1 [61], Retinopathy Online Challenge (ROC) [62], MESSIDOR [63], High resolution fundus (HRF) [64], E-Optha [65], Hamilton Eye Institute Macular Edema Dataset (HEI-MED) [66], Kaggle Diabetic Retinopathy dataset [67], Retinal Vessel Image set for Estimation of Widths (REVIEW) [68] and private databases. Thresholding, morphological operations, DR grading, classification (Supervised or Unsupervised) and wavelets are mostly used in many works. The virtues and flaws of these methods are reviewed in the literature and will be helpful to researchers to plan future work on DR detection as per their specific objective.

\section{REFERENCES}

1. The International Agency for the Prevention of Blindness (IAPB). Diabetic Retinopathy. [Online].

2. Fong DS, Aiello L, Gardner TW, et al. Retinopathy in diabetes. Diabetes Care 2004; 27 Suppl 1: S84-87.

3. R.Ravindraiah and S.Chandra Mohan Reddy. An Automated Exudate detection in Diabetic Retinopathy fundus images using Multi Kernel Spatial Fuzzy C means clustering method, International Journal of Engineering and Technology (UAE), Vol 7 (1.8), pp 10-14, Feb 2018, ISSN: 2227-524X.

4. R.Ravindraiah and S.Chandra Mohan Reddy. Exudates detection in Diabetic Retinopathy images using Possibilistic $\mathrm{C}$ means clustering algorithm with induced spatial constraint, Advances in Intelligent Systems and Computing, Springer Nature Series, Singapore, pp 455-463, March 2018.

5. R.C.Gonzalez and R.E. Wood, "Digital image processing using Matlab", Pearson Education Inc. and Dorling Kindersley Publishing Inc.

6. R.P. Phillips, J. Forrester and P. Sharp. Automated detection and quantification of retinal exudates, Graefe Archive for Clinical Experimental Ophthalmology, 231, pp 90-94, 1993.

7. G. G. Gardner, D. Keating, T. H. Williamson and A. T. Elliott. Automatic detection of diabetic retinopathy using an artificial neural network: a screening tool, $\mathrm{Br} \mathrm{J}$ Ophthalmol 80 (1996) pp 940-944.

8. Ege $\mathrm{B}$, Larsen $\mathrm{O}$ and Hejlesen $\mathrm{O}$. Detection of abnormalities in retinal images using digital image analysis. 1999;8 pp 33-40.

9. H. Wang, W. Hsu, K. Goh and M. Lee. An effective approach to detect lesions in colour retinal images, in Proceedings of the IEEE Conference on Computer Vision and Pattern Recognition, Vol. 2, pp. 181-187 2000.

10.C. Sinthanayothin, J.F. Boyce, T.H. Williamson and H.L. Cook. Automated Detection of Diabetic Retinopathy on Digital Fundus Image, International Journal of Diabetic Medicine, vol. 19, pp. 105-112, 2002.

11.T. Walter, J. C. Klein, P. Massin, and A. Erginay. A Contribution of Image Processing to the Diagnosis of Diabetic Retinopathy - Detection of Exudates in Color 
Fundus Images of the Human Retina, IEEE Trans. Med.Imag., Vol. 21, No. 10, pp. 1236-1243, 2002.

12. A. Osareh, M. Mirmehdi, B. T. Thomas and R. Markham, Classification and localisation of diabetic-related eye disease, in: Proceedings of the 7th European Conference on Computer Vision-Part IV, ECCV '02, 2002.

13.C. Sinthanayothin, V. Kongbunkiat, S Phoojaruenchanachai and A. Singalavanija, Automated screening system for diabetic retinopathy: Image and Signal Processing and Analysis. Proceedings of the 3rd International Symposium on, Vol. 2, 2003, pp. 915 _ 920.

14. Osareh, M. Mirmehdi, B. Thomas and R. Markham, Automated identification of diabetic retinal exudates in digital colour images, Br J Ophthalmol 87 (2003) pp. 1220-1223.

15. C. I. Sanchez, R. Hornero, M. I. Lopez and J. Poza, Retinal image analysis to detect and quantify lesions associated with diabetic retinopathy, in: Engineering in Medicine and Biology Society, 26th Annual International Conference of the IEEE, Vol. 1, 2004, pp. 1624_1627.

16. Usher D, Dumskyj M, Himaga M, Williamson TH, Nussey $\mathrm{S}$ and Boyce J, "Automated detection of diabetic retinopathy in digital retinal images:a tool for diabetic retinopathy screening, Diabet Med. 2004 Jan;21(1):84-90.

17.E. Grisan and A. Ruggeri. A hierarchical Bayesian classification for non-vascular lesions detection in fundus images, in IFMBE Proc, 2005, pp. 1727-1983.

18.X. Zhang and O. Chutatape. Top-down and bottom up strategies in lesion detection of background diabetic retinopathy, in IEEE Conference on Computer Vision and Pattern Recognition. Proceedings. IEEE, 2005, vol. 2, pp. 422-428.

19. Alan D Fleming, Sam Philip, Keith A Goatman, Graeme J Williams, John A Olson and Peter F Sharp, Automated detection of exudates for diabetic retinopathy screening. Physics In Medicine And Biology 52 (2007) 7385-7396

20. Meindert Niemeijer, Bram van Ginneken, Stephen R. Russell and Maria S. A. Suttorp-Schulten and Michael D. Abra`moff, Automated Detection and Differentiation of Drusen, Exudates, and Cotton-Wool Spots in Digital Color Fundus Photographs for Diabetic Retinopathy Diagnosis, IOVS, May 2007, Vol. 48, No. 5, pp 2260 2267

21. Giri Babu Kande, P.Venkata Subbaiah and T.Satya Savithri. Segmentation of Exudates and Optic Disc in Retinal Images, 6th Indian Conference on Computer Vision, Graphics \& Image Processing, 2008

22. Sanchez C.I, Horneo R, Lopez M.I, Aboy M, Poza J and Abasolo D. A novel automatic image processing algorithm for detection of hard exudates based on retinal image analysis, Medical Engineering Physics, vol. 30, no. 3, pp 35, 2008

23. U. R. Acharya, K. C. Chua, E. Y. K. Ng, W. Yu and C. Chee, Application of higher order spectra for the identication of diabetes retinopathy stages, Journal of Medical Systems 32 (2008), pp 481-488.

24. Sopharak, et al. Automatic detection of diabetic retinopathy exudates from nondilated retinal images using mathematical morphology methods, Computerized medical imaging and graphics, vol. 32, pp. 720-727, 2008.

25. Sopharak, K. Thet Nwe, Y. A. Moe, M. N. Dailey and B. Uyyanonvara. Automatic exudate detection with a naïve bayes classifier. In International Conference on Embedded Systems and Intelligent Technology (ICESIT), pages 139-142, 2008.

26. J. Suthammanas, K. Viriyachot, P. Tuntipark, B. Uyyanonvara, K. Krairaksaand C. Duanggate, An automatic diabetic retinopathy tele screening system of thailand, in: Proceedings of the International Symposium on Biomedical Engineering (ISBME 2008), 2008, pp. 88_92.

27. U. R. Acharya, C. M. Lim, E. Y. K. Ng, C. Chee and T. Tamura, Computer-based detection of diabetes retinopathy stages using digital fundus images, Proceedings of the Institution of Mechanical Engineers, Part H: Journal of Engineering in Medicine 223 (5) (2009) 545_553.

28. A. Sopharak, B. Uyyanonvara, and S. Barman. Automatic Exudate Detection from Non-dilated Diabetic Retinopathy Retinal Images Using Fuzzy C-means Clustering, Sensors, pp. 2148-2161, 2009.

29. Maria Gracia, Clara 1. Sanchez, Maria 1. Lopez, Daniel Abcisolo, and Roberto Homero, Neural network based detection of hard exudates in retinal images, Computer Methods and Programs in Biomedicine, vol. 93, no. 1, pp. 9-19, 2009

30. M. García, C. Sánchez, J. Poza, M. López, and R. Hornero. Detection of Hard Exudates in Retinal Images Using a Radial Basis Function Classifier, Annals of Biomedical Engineering, vol. 37, pp. 1448-1463, 2009.

31. Sopharak, B. Uyyanonvara, and S. Barman, Automatic exudate detection for diabetic retinopathy screening, Science Asia, vol. 35, pp. 80-88, 2009. doi: 10.2306/scienceasia1513-1874.2009.35.080

32.C. I. Sánchez, M. García, A. Mayo, M. I. López, and R. Hornero. Retinal image analysis based on mixture models to detect hard exudates, Medical Image Analysis, vol. 13, pp. 650-658, 2009.

33. Alireza Osareh, Bita Shadgar, and Richard Markham, A Computational-Intelligence-Based Approach for Detection of Exudates in Diabetic Retinopathy Images, IEEE Transactions on Information Technology in Biomedicine, Vol. 13, No. 4, July 2009, Pp 535-545

34. Akara Sopharak, Bunyarit Uyyanonvara and Sarah Barman Automatic Exudate Detection from Non-dilated Diabetic Retinopathy Retinal Images Using Fuzzy C-means Clustering, Sensors 2009, 9, pp 2148-2161; doi: $10.3390 /$ s90302148

35.D. Welfer, J.Scharcanski and D.R.Marinho. A coarse-to-fine strategy for automatically detecting exudates in color eye fundus images, Comput.Med. Imaging Graph. 34(3) (2010), pp 228-235.

36. Sopharak et.al.,A. Fine exudate detection using morphological reconstruction enhancement, Applied biomedical engineering, vol. 1, pp. 45-50, 2010.

37. B. Dupas, T.Walter, A.Erginay, R.Ordonez, N.Deb-Joardar, P.Gain, J.Klein, and Massin, Evaluation of automated fundus photograph analysis algorithms for detecting microaneurysms, haemorrhages and exudates, and of a computer- assisted diagnostic system for grading diabetic retinopathy, Diabetes Metab. 36 (2010)213-220.

38.C.Sanchez, M.Niemeijer, S.Schulten, M.Abramo and B.vanGinneken: Improving hard exudate detection in retinal images through a combination of local and contextual information, in: Proceedings of IEEE International Symposium on Biomedical Imaging, 2010,pp 5-8.

39. C. Agurto, V. Murray, E. Barriga, S. Murillo, M. Pattichis, H. Davis, S. Russell, M. Abramo and P. Soliz, Multiscale AM-FM methods for diabetic retinopathy lesion detection, Medical Imaging, IEEE Transactions on 29 (2) (2010) pp 502-512.

40.M. D. Abràmo, J. M. Reinhardt, S. R. Russell, J. C. Folk, V. 
B. Mahajan, M. Niemeijer and G. Quellec, Automated early detection of diabetic retinopathy, Ophthalmology 117 (6) (2010), pp 1147-1154.

41.C. Sinthanayothin et. al. Image analysis for automatic diagnosis of Diabetic Retinopathy, Journal of Medical Science, Vol. 35,No. 5, pp. 1491- 1501, Jan 2011

42.S. Kavitha, K. Duraiswamy, Kavitha, S and Duraiswamy, $\mathrm{K}$. Automatic detection of hard and soft exudates in fundus images using color histogram thresholding, European Journal of Scientific Research. (2011), 48, pp 493-504.

43. M. U. Akram and S. A. Khan, Automated detection of dark and bright lesions in retinal images for early detection of diabetic retinopathy, J. Med. Syst., doi:10.1007/s10916-011- 9802-2, 2011

44. Rocha, T. Carvalho, H. F. Jelinek, S. Goldenstein, and J. Wainer. Points of interest and visual dictionaries for automatic retinal lesion detection, Biomedical Engineering, IEEE Transactions on, vol. 59, pp. 2244-2253, 2012.

45. S. Ali, D. Sidibé, K. M. Adal, L. Giancardo, E. Chaum and T. P. Karnowski, et al., Statistical atlas based exudate segmentation, Computerized Medical Imaging and Graphics, vol. 37, pp. 358-368, 7// 2013.

46.B.Harangi, I.Lazar and A.Hajdu, Automatic exudate detection using active contour model and region wise classification, in: 34th Annual International Conference of the IEEEE MBS, SanDiego, California USA, 2012, pp.5951-5954.

47.B.Harangi and A.Hajdu, Improving automatic exudate detection based on the fusion of the results of multiple active contours in: IEEE 10th International Symposium on Biomedical Imaging (ISBI), 2013, pp.45-48.

48. B Harangi and A. Hajdu , Automatic exudate detection by fusing multiple active contours and region wise classification, Computers in Biology and Medicine 54 (2014) 156-171

49.X. Zhang, G. Thibault, E. Decencière, B. Marcotegui, B. Laÿ, R. Danno, et al. Exudate detection in color retinal images for mass screening of diabetic retinopathy,Medical Image Analysis, vol. 18, pp. 1026-1043, 2014.

50.C. Pereira, L. Gonçalves, and M. Ferreira. Exudate segmentation in fundus images using an ant colony optimization approach, Information Sciences, vol. 296, pp. 14-24, 2015.

51. Jyoti Prakash Medhi $\mathrm{N}$ et.al. An effective fovea detection and automatic assessment of diabetic maculopathy in color fundus images", Computers in Biology and Medicine 74 (2016) pp 30-44.

52.B. Harangi and A. Hajdu. Automatic exudate detection by fusing multiple active contours and region wise classification, Computers in Biology and Medicine 54 (2014) pp 156-171.

53. Elaheh Imani, Hamid-Reza Pourreza, A novel method for retinal exudate segmentation using signal separation algorithm, Computer Methods and Programs in Biomedicine $\quad \mathrm{http} / / / \mathrm{dx}$.doi.org/doi: 10.1016/j.cmpb.2016.05.016

54. Shuang Yu, Di Xiao and Yogesan Kanagasingam, Exudate Detection for Diabetic Retinopathy With Convolutional Neural Networks, 2017 IEEE, pp 1744- 1747

55. Sudeshna Sil Kar and Santi P. Maity, Automatic Detection of Retinal Lesions for Screening of Diabetic Retinopathy, IEEE Transactions on Biomedical Engineering, 2018, pp $1-9$.

56. Sudeshna Sil Kar and Santi P. Maity. Gradation of diabetic retinopathy on reconstructed image using compressed sensing, IET Journal of Image Processing, 2018, Vol. 12 Iss. 11, pp. 1956-1963.
57.Zhentao Gao, Jie Li, Jixiang Guo, Yuanyuan Chen, Zhang $\mathrm{Yi}$ and Jie zhong, Diagnosis of Diabetic Retinopathy Using Deep Neural Networks, IEEE Access, Vol 7, pp 3360-3370,2019.

58. J. Staal, M. D. Abramoff, M. Niemeijer, M. A. Viergever, and B. van Ginneken. Ridge-based vessel segmentation in color images of the retina, IEEE Trans. Med. Imag., vol. 23, no. 4, pp. 501-509, 2004.

59.D. Hoover, V. Kouznetsova, and M. Goldbaum. Locating blood vessels in retinal images by piecewise threshold probing of a matched filter response, IEEE Trans. Med. Imag, 19 (3), pp. 203-210, 2000.

60.T. Kauppi et al., DIARETDB0: Evaluation database and methodology for diabetic retinopathy algorithms, Lappeenranta Univ. Technol., Lappeenranta, Finland, Tech. Rep., 2006.

61. T. Kauppi, V. Kalesnykiene, J.-K. Kamarainen, L. Lensu, I. Sorri, and A. Raninen, DIARETDB1 diabetic retinopathy database and evaluation protocol, in Proc. 11th Conf. Med Image Understand. Anal., Aberystwyth, Wales, Jul. 2007, pp. 61-65.

62. Retinopathy Online Challenge (ROC) Database (December 12, 2015), http://webeye.ophth.uiowa.edu/ROC/

63.E. Decencière et al., Feedback on a publicly distributed image database: The messidor database, Image Anal. Stereology, vol. 33, no. 3, pp. 231-234, 2014. [Online].

64. High Resolution Fundus (HRF) Image Database (December 12 , 2015), https://www5.cs.fau.de/research/data/fundus-images/

65.E. Decencière et al. TeleOphta: Machine learning and image processing methods for tele ophthalmology, IRBM, vol. 34, no. 2, pp. 196-203, 2013. [Online]. Available: http://www.sciencedirect.com/science/article/pii/S1959-0 318(13)00023-7

66. Giancardo, L, Meriaudeau, F, Karnowski, T. P, Li, Y, Garg, S, Tobin, Jr, K. W and Chaum, E. 'Exudate-based diabetic macular edema detection in fundus images using publicly available datasets, Medical Image Analysis 16(1), 216-226, (2012) https://www.iasiss.org/ojs/IAS/article/view/1155

67. Diabetic Retinopathy Detection. Accessed: Sep. 1, 2017. [Online].

Available: https://www.kaggle.com/c/diabetic-retinopathy-detection /data

68. B. Al-Diri, A. Hunter, D. Steel, M. Habib, T. Hudaib, and S. Berry, REVIEW-A reference data set for retinal vessel profiles, in Proc. 30th Annu. Int. Conf. IEEE Eng. Med. Biol. Soc., Aug. 2008, pp. 2262-2265. 